\title{
An Approach to Visualize the Deformation of the Intermediate Filament Cytoskeleton in Response to Locally Applied Forces
}

\author{
Jiashan Wang and Andrew E. Pelling \\ Department of Physics and Department of Biology, University of Ottawa, MacDonald Hall,150 Louis Pasteur, Ottawa, \\ ON, Canada K1N 6N5 \\ Correspondence should be addressed to Andrew E. Pelling, a@pellinglab.net
}

Received 28 August 2011; Accepted 25 September 2011

Academic Editors: P. Lavia and C. C. Uphoff

Copyright (๑) 2012 J. Wang and A. E. Pelling. This is an open access article distributed under the Creative Commons Attribution License, which permits unrestricted use, distribution, and reproduction in any medium, provided the original work is properly cited.

\begin{abstract}
The intermediate filament (IF) cytoskeleton plays an important role in integrating biomechanical pathways associated with the actin and microtubule cytoskeleton. Vimentin is a type III IF protein commonly found in fibroblast cells and plays a role in transmitting forces through the cytoskeleton. Employing simultaneous laser scanning confocal and atomic force microscopy (AFM), we developed a methodology to quantify the deformation of the GFP-vimentin-labeled IF cytoskeleton as a function of time in response to force application by the AFM. Over short times (seconds), IFs deformed rapidly and transmitted force throughout the entire cell in a highly complex and anisotropic fashion. After several minutes, mechanically induced displacements of IFs resemble basal movements. In well-adhered cells the deformation of IFs is highly anisotropic as they tend to deform away from the longitudinal axis of the cell. This study demonstrates that simultaneous AFM and LSCM can be employed to track the deformation and dissipation of force through the IF cytoskeleton.
\end{abstract}

\section{Introduction}

Recent advancements in the field of biophysics, such as the rapid improvement of our understanding of the mechanical roles of the cytoskeleton (CSK) and extracellular matrix (ECM) [1], have led to a picture of the cell from a mechanical point of view, as opposed to the traditional biochemical perspective [1-3]. It is now known that biological processes such as DNA replication are not only affected by the presence of certain biochemical signals in the cell, but also by mechanical forces such as tension on the DNA strand itself [4]. The new perspective involving physical forces at a micro- and nanoscale has allowed for many new discoveries [1]. For one, the process of mechanotransduction occurs by changes in the concentrations of local signaling molecules as a response to the deformation of the CSK induced by externally applied forces [5]. This process depends on the close integration of the three cytoskeletal elements, actin, microtubules (MTs), and intermediate filaments (IFs) [1]. Understanding how these cytoskeletal elements deform will provide insight into how cells both sense and react to their external physical environment, which results in the conversion of mechanical signals from the ECM to the CSK and eventually into cellular signaling $[1,2]$.

The IF network is one of the three main components of the CSK. However, the intermediate filament network has since been proven to play a major role in the mechanical functions of the cell [6-8]. IFs have been shown to stabilize MTs against compressive forces and while supported by IFs, MT filaments resistance to buckling under compression may increase by orders of magnitude [9]. IFs also serve as a vital connection between the CSK, the extracellular matrix (ECM), and the nucleoskeleton. The IF CSK connects the CSK to the ECM through desmoplakins and integrins [10]; at the same time, it has been shown to connect the CSK to the nucleoskeleton via interactions between $\beta$-lamins and vimentin, a class III intermediate filament protein [11]. Computational modeling has shown that cells with higher IF density display increased deformation of the nucleus in response to the external force [6]. Such transmission of forces from the exterior to the nucleus is suspected to play a role in the mechanism of mechanotransduction [12]. 


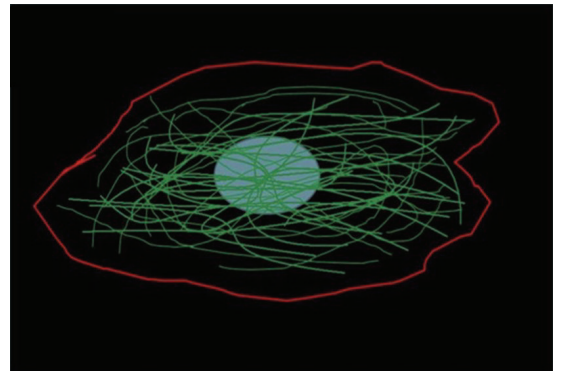

(a)

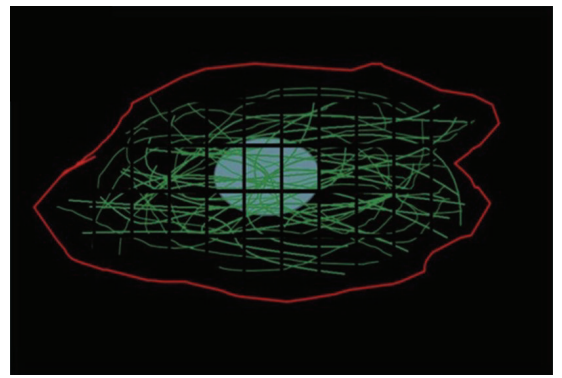

(b)

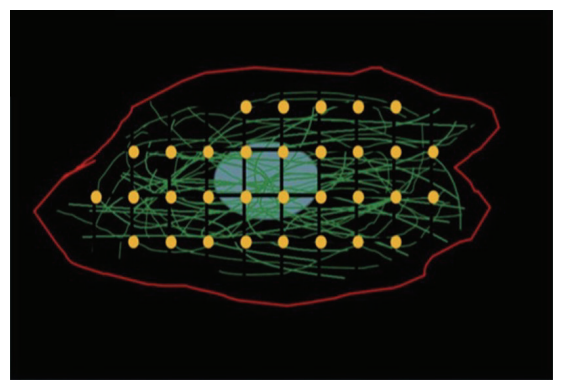

(c)

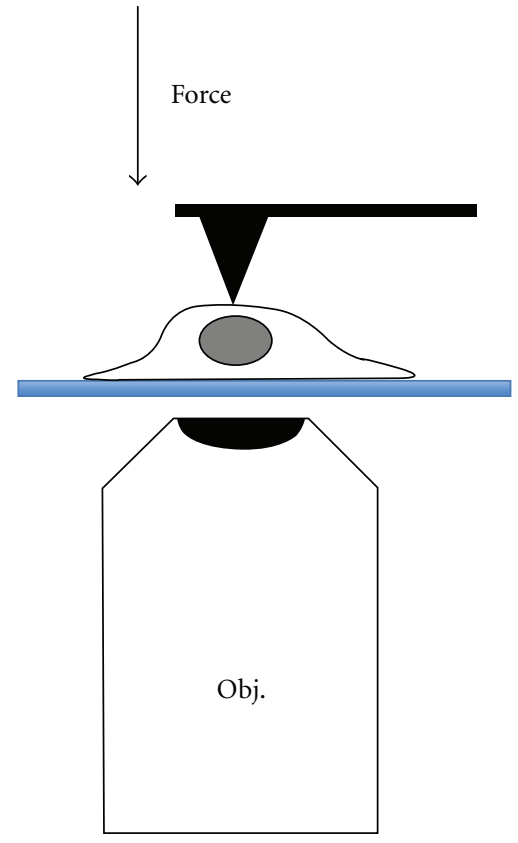

(d)

FIGURE 1: Schematic of the experimental setup. (a) A cell transiently expressing GFP-Vimentin is located. (b) A grid (3.2 $\mu \mathrm{m}$ spacing with $0.5 \mu \mathrm{m}$ lines) is photobleached into the IF CSK. (c) The nodes (yellow dots) of the grid are tracked over time. (d) Side view of the combined AFM-LSCM. An AFM cantilever is used to apply a constant downward mechanical force to the centre of the nucleus in a cell expressing GFP-vimentin. Simultaneous LSCM is employed to image the deformation of the IF CSK on an inverted microscope.

The IF network has unique properties that enable it to fulfill its role in the mechanical structure of the cell [13]. Unlike the polar, globular subunits of actin and MTs, IFs are made of neutral subunits of $\alpha$-helical polypeptide chains. Due to their differing composition, IFs respond differently from actin and MTs to physical stress, defined as the amount of force applied over a given area. The IF network is able to maintain integrity at strains of up to $100 \%$ whereas the actin and MT networks rupture at $20 \%$ and $60 \%$, respectively [13]. IFs are also unique in their nonlinear resistance to deformation. IFs show very little resistance to deformation at low strains, but at higher strains (towards 100\%), the resistance to further deformation sharply increases [14]. In contrast, the actin CSK is very resistant to deformation at low strains and is quickly able to recover initial conformation after long periods of strain, but rapidly depolymerizes when the network is stretched over $20 \%$ of its original dimensions [13]. The overall picture of IF mechanics emerges as being highly malleable at physiological conditions, but highly resistant against deformation when large applied forces create large strains in the CSK [15]. It is this nonlinear response to physical strain that allows IFs to provide a safe barrier against overextension of the cell structure [15] by dramatically increasing the CSK's stiffness when strain of the CSK exceeds the limits of the actin and MT components [6]. Due to resilience of the IF CSK at a wide range of forces, the IF network is able to transmit forces applied on the focal adhesions (FAs) from the exterior of the cell to the nucleus effectively across a wide range of forces [12], whereas the actin network is only able to effectively transmit forces to the nucleus at low ranges, as it is likely to depolymerize as the applied force increases [12].

An important tool for studying the mechanical properties of the cell is the atomic force microscope (AFM). The AFM was invented in 1986 as a scanning probe microscope (SPM) for the purposes of imaging [16] and has since been applied to biology for both imaging and measuring the mechanical properties of living cells [17-21]. Further insight into the mechanics of the CSK may be studied by the integration of AFM with optical imaging systems such as the 
fluorescence microscope [19, 22]. Lehenkari et al. designed a system by which the AFM can be mounted on top of the stage of an inverted optical microscope, allowing for simultaneous optical/AFM microscopy [22]. This is crucial in allowing the observation of real-time responses of the CSK to mechanical stimuli, as opposed to fixing and staining the sample after force measurements using the AFM [17]. The technology of AFM/optical microscopy integration has opened the door to observing the immediate response of the CSK to an external force applied by the AFM, such that the transmission of force through the CSK, which often occurs within the seconds to minutes after a mechanical stimulus [21]. This method of visualizing the CSK using laser scanning confocal microscopy (LSCM) while applying external force with the AFM will be used in our study to observe the immediate response of the vimentin CSK to indentation by the AFM.

The goal of the study was to establish a methodology utilizing simultaneous AFM-LSCM to observe and quantify the deformation of the complex IF CSK network in response to locally applied forces. This was accomplished by creating cells transiently expressing a GFP-vimentin-labeled IF CSK. We then nondestructively photobleached a well-defined grid pattern into the IF CSK [23]. Once the grid was created, the cell was imaged for several minutes under an applied load from the AFM or under no load. The deformation of the IF CSK was determined by tracking the movement of the nodes of the photobleached grid. The average displacement magnitudes of the nodes of control and indented cells were found to be significantly different immediately following the indentation. However, over time the basal average displacement magnitude of the IFs was similar to the mechanically stimulated cells. This reveals the constant dynamic movement of the IF network even in the absence of externally applied forces [12]. Locally, we found that the deformation of the IF CSK appears to be anisotropic and that nodes near the central longitudinal axis of the cell show little displacement, whereas increasing displacement occurred further away from the central longitudinal axis. The IF CSK is considered the main cytoskeletal element that acts to transmit mechanical forces from the extracellular matrix into the nucleus through its interactions with vinculin and actin [12]. Therefore, understanding the deformation of the IF CSK may shed light on the mechanism of mechanotransduction, bringing a better understanding of the exact process by which cells incorporate mechanical signals from the external physical environment into biological processes.

\section{Materials and Methods}

2.1. Mammalian Cell Culture and Transfections. NIH3T3 mouse fibroblast cells were cultured in High glucose Dulbecco Modified Eagle's Medium (DMEM) containing 10\% Fetal Bovine Serum (FBS) and 1\% penicillin/streptomycin antibiotics (all from Hyclone). The cells were cultured at $37^{\circ} \mathrm{C}$ at $5 \% \mathrm{CO}_{2}$ in $10 \mathrm{~cm}$ diameter plastic working plates. Cells were passaged every 4 days and split into $35 \mathrm{~mm}$ glass bottom dishes (MatTek) at 5000 cells per dish to ensure evenly spaced single cells for experiments. GFP-vimentin DNA plasmids (a kind gift of Robert Goldman, Northwester

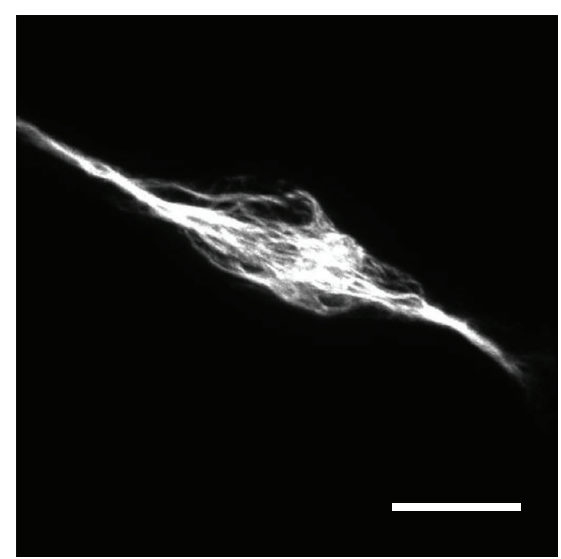

(a)

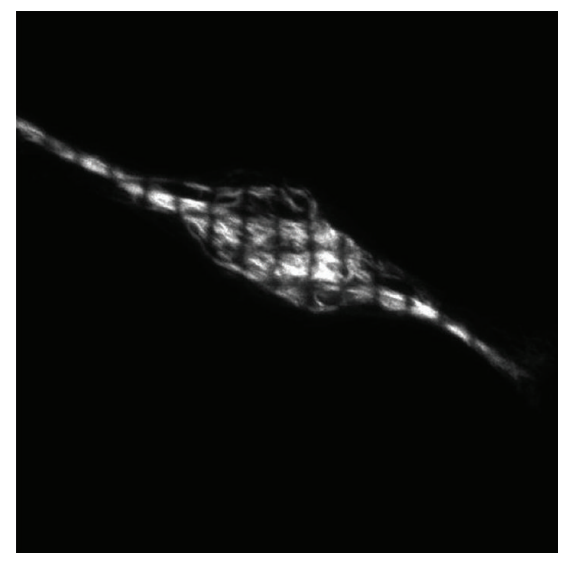

(b)

Figure 2: Fibroblast cells transfected with GFP-vimentin-labeled IFs. (a) A cell expressing GFP IFs before photobleaching. (b) The IFs after photobleaching a grid pattern using the LSCM (Scale bar = $10 \mu \mathrm{m})$.

University) were transfected using lipofectamine 2000 (Invitrogen) according to manufacturer protocols. The cells were incubated at $37^{\circ} \mathrm{C}$ overnight, and experiments were carried out the next day.

2.2. Immunofluorescence Labeling. GFP-Vimentin expressing NIH3T3 cells were fixed using 3.5\% paraformaldehyde and $2 \%$ sucrose at $37^{\circ} \mathrm{C}$ and permeabilized using $0.5 \%$ Triton $\mathrm{X}-100$ at $37^{\circ} \mathrm{C}$. Actin was labeled by incubating the sample for $2 \%$ phalloidin conjugated to AlexaFluor 546 fluorophore (Invitrogen), followed by incubation with wash buffer (phosphate buffered saline (PBS) containing 5\% FBS and 0.05\% Sodium Azide). Vinculin was labeled using 0.5\% monoclonal anti-vinculin primary antibody produced in mouse (Sigma) and $0.5 \%$ rabbit anti-mouse IgG secondary antibody conjugated to AlexaFluor 546 fluorophore. DNA was labeled with 0.5\% DAPI (Invitrogen) [21].

2.3. Laser Scanning Confocal Microscopy (LSCM). Both imaging and photobleaching were performed with a Nikon A1R high-speed LSCM on a Nikon TiE optical microscope 
Vinculin

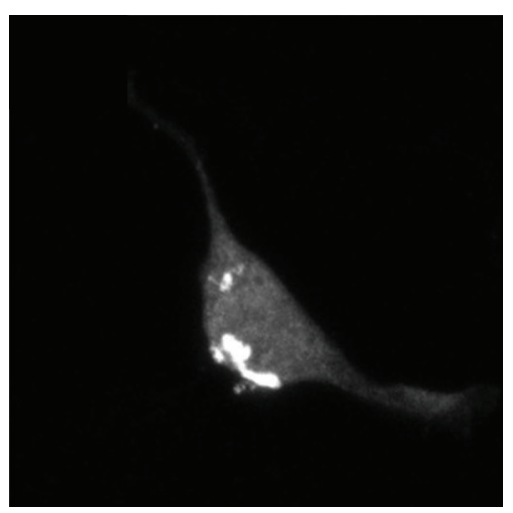

Vinculin

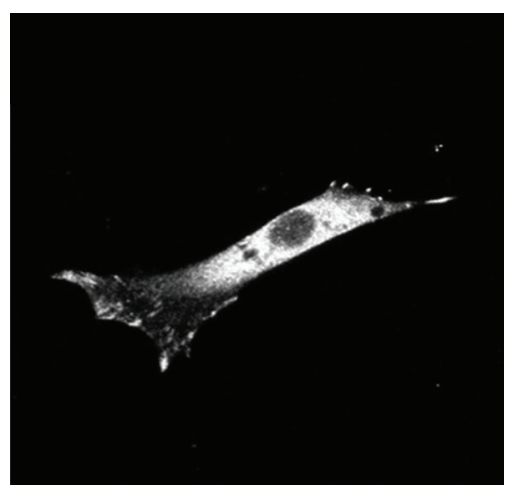

Vimentin

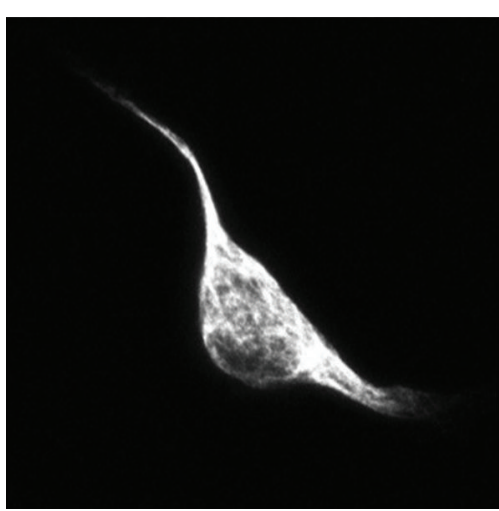

(a)

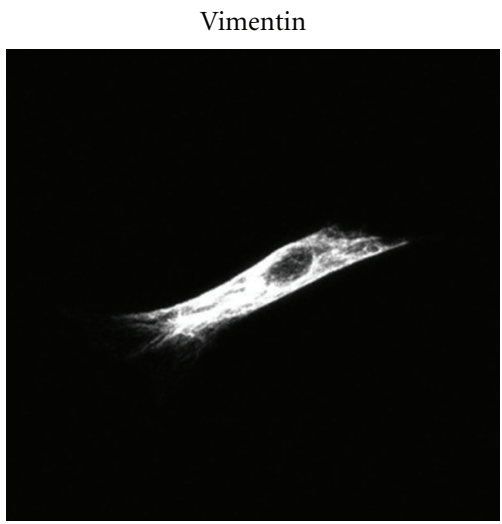

(b)
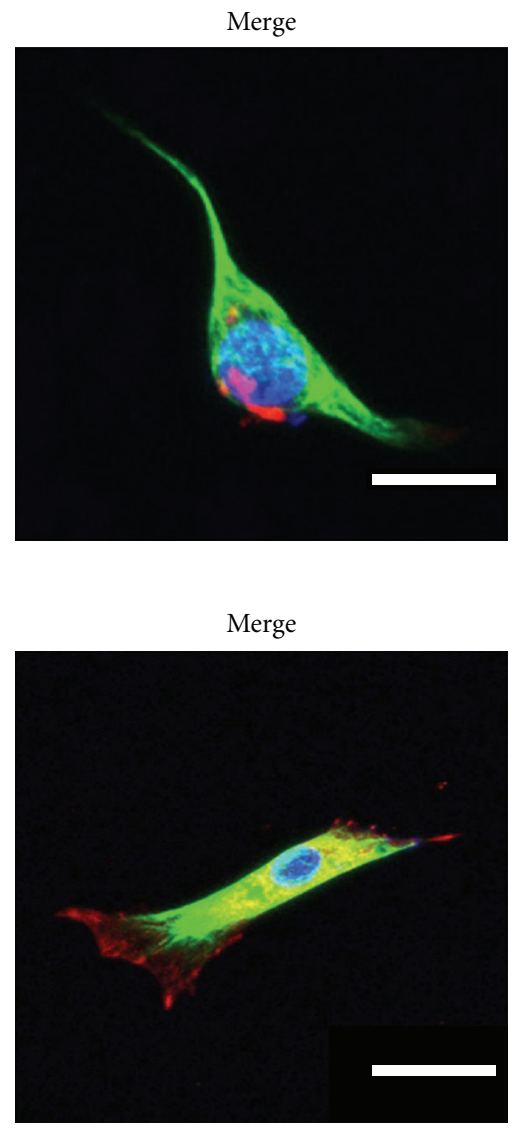

FIGURE 3: Images of focal adhesions (vinculin) and vimentin. Elongated (a) and flat (b) NIH3T3 mouse fibroblasts stained for vinculin and transfected with GFP-vimentin. RGB merged images show vinculin in red, vimentin in green, and DNA in blue. Scale bar $=10 \mu \mathrm{m}(\mathrm{a}), 20 \mu \mathrm{m}$ (b).

platform (Nikon, Canada). Live cells containing GFP-labeled IFs were imaged using a $63 \mathrm{x}$ oil immersion objective. Confocal volumes consisting of $1 \mu \mathrm{m}$ slices were acquired every $30 \mathrm{sec}$; all images for analysis were maximum intensity projections. Photobleaching (Figures $1(\mathrm{a})-1(\mathrm{c})$ ) of a userdefined grid pattern was achieved with a $488 \mathrm{~nm}$ laser at full power at multiple focal planes in the confocal volume. The grid had a distance between lines of $3.2 \mu \mathrm{m}$, and the lines in the grid had thicknesses of $\sim 0.5 \mu \mathrm{m}$.

2.4. Atomic Force Microscope (AFM). All force-distance curves and nanoindentations were performed using the JPK Nanowizard II AFM head (JPK) mounted on the microscope described above (Figure 1(d)). AFM tips had an experimentally determined average spring constant of $67 \pm 8 \mathrm{pN} / \mathrm{nm}$ (Nanoworld). Cells were indented at the center of the nucleus with a constant $20 \mathrm{nN}$ of force for a total of 4 mins during which LSCM imaging was carried out as described above. To determine the average cellular elasticity (or Young's modulus), five force curves were measured per cell ( $n=10$ cells) and analyzed using the Hertz indentation model for the shallow indentation $(200 \mathrm{~nm})$ of a stiff cone into a soft substrate [24].
2.5. Data Analysis. Nodes of the photobleached grid were tracked through time using the MTrackJ add-on for ImageJ (http://imagej.nih.gov/ij/). The results were plotted as a displacement magnitude contour map in Origin (OriginLab) and the location of the AFM indentation marked with an " $x$ ". The average displacements of cells for both control and indented experiments were plotted individually as a function of time in scatter plots as average \pm standard error. Statistical difference between the average displacements between cells of control and indented experiments was calculated with Student's $t$-test $(\alpha=0.05)$ for each time point.

\section{Results}

The transient transfection of GFP-vimentin produced a visible IF CSK (Figure 2(a)). After photobleaching, a $16 \times$ 16 grid was produced in the IF CSK (Figure 2(b)), which remained visible throughout the duration of the experiment. After transfection, the majority of cells had flat fibroblastic morphology with IFs well spread throughout the cell body ("flat cells"). However, a subpopulation of cells existed with a distinct elongated and spindle-like morphology ("elongated cells"), with a marked increase in cell height (xx um versus 
Actin

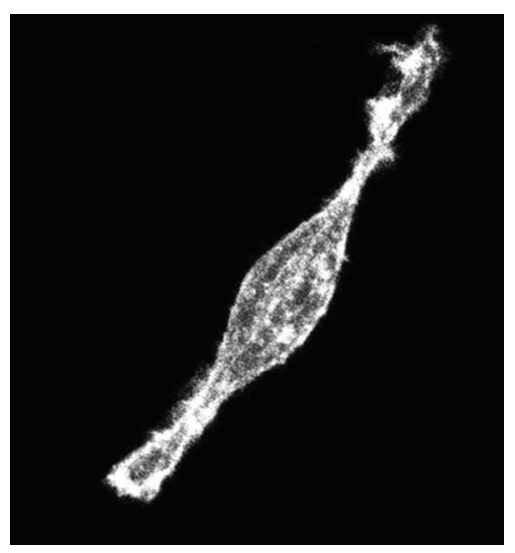

Actin

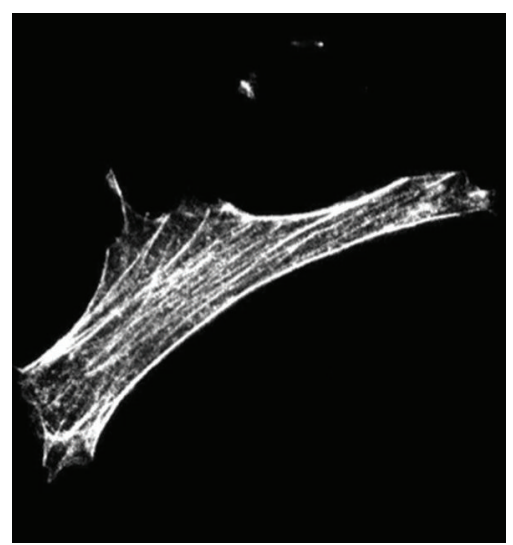

Vimentin

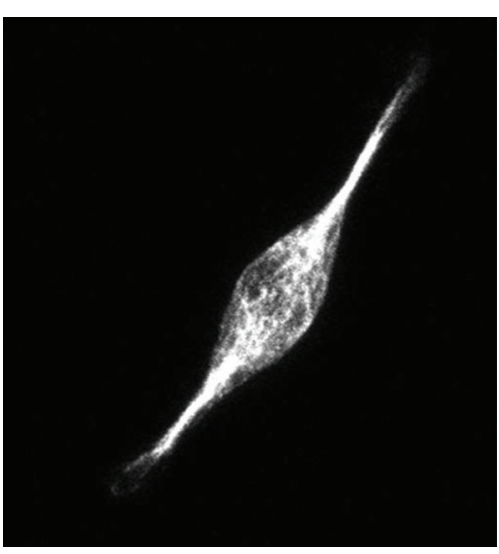

(a)

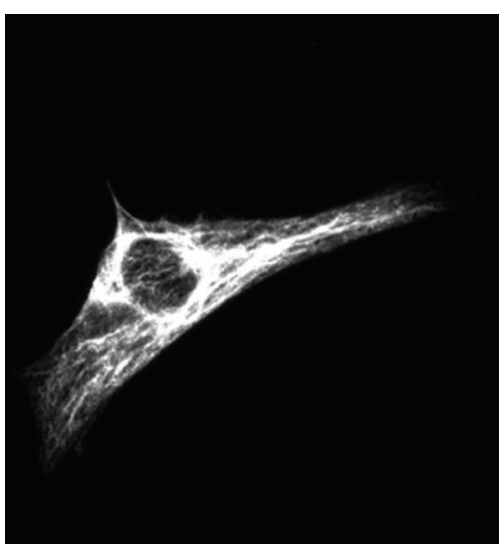

(b)

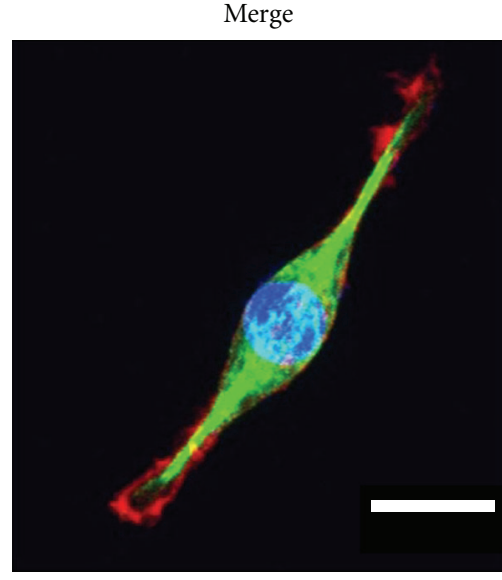

Merge

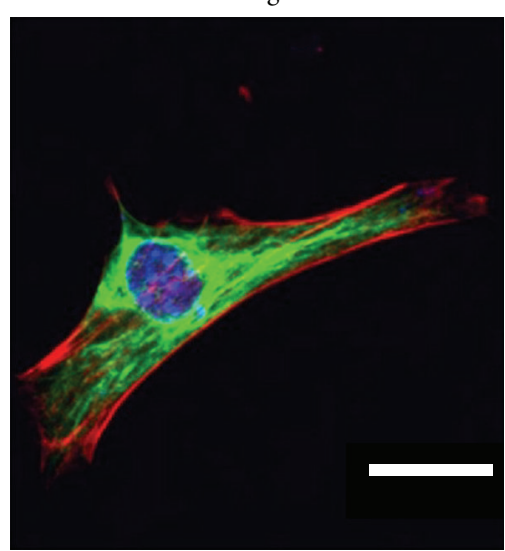

FIgURE 4: Images of the actin and IF CSK. Elongated (a) and flat (b) NIH3T3 mouse fibroblasts stained for actin and transfected with GFP-vimentin. RGB merged images show actin in red, vimentin in green, and DNA in blue. Scale bar $=10 \mu \mathrm{m}(\mathrm{a}), 15 \mu \mathrm{m}(\mathrm{b})$.

yy um). The IF CSK in these cells appeared collapsed around the nucleus and forming spindle-like protrusions. We also investigated FA structure as they provide a qualitative picture of how well adhered a cell is to the substrate. VimentinGFP-transfected cells fixed and stained for vinculin-rich FAs (Figure 3), reveal that elongated cells (Figure 3(a)) had fewer FAs than flat cells (Figure 3(b)). This results in an inability to transmit forces from the FAs into the CSK [25]. It has also been postulated that fibroblast cells of this morphology have lower substrate-adhesion (which is consistent with our study) [26]. Differences were also noted in the actin CSK (Figure 4), where the actin network forms clear stress fibres in flat cells (Figure 4(b)) but is mostly depolymerized in the elongated cells (Figure 4(a)). It is interesting to note, however, that the IF CSK remains intact in both cases. Furthermore, elongated and flat cells are also mechanically distinct as they have an average Young's moduli of $3.3 \pm$ 0.1 and $5.9 \pm 0.2 \mathrm{kPa}$, respectively (Figure 5(a)). Although the exact physiological state of the elongated cells is not known, their differences in mechanics and morphology make an interesting comparison to the typical morphologies of the flat cells. Importantly, the elongated morphology of the fibroblasts in this study is more typical of fibroblasts found in primary tissue samples [27] and there continues to be a great deal of debate as to which morphology is representative of a "normal" fibroblast. Therefore, we chose to examine both morphologies using our methodology to identify difference in their ability to transmit applied mechanical forces.

As described above, flat and elongated cells were indented using the AFM for 4 minutes, imaged every 30 seconds, and the resulting displacement of the nodes in the photobleached grid in the vimentin CSK was tracked (control: $n=3$ elongated cells and $n=3$ flat cells; mechanically stimulated: $n=3$ elongated cells and $n=3$ flat cells). The control cells were imaged and tracked in the same manner but were not indented. The average grid node displacements were calculated and graphed as a function of time (Figure 5(b)). Overall, the displacement of the IFs in elongated cells was significantly higher than that in flat cells for both control and mechanically stimulated cells.

Elongated and flat control cells revealed that the natural movement of IFs away from their initial positions is relatively 


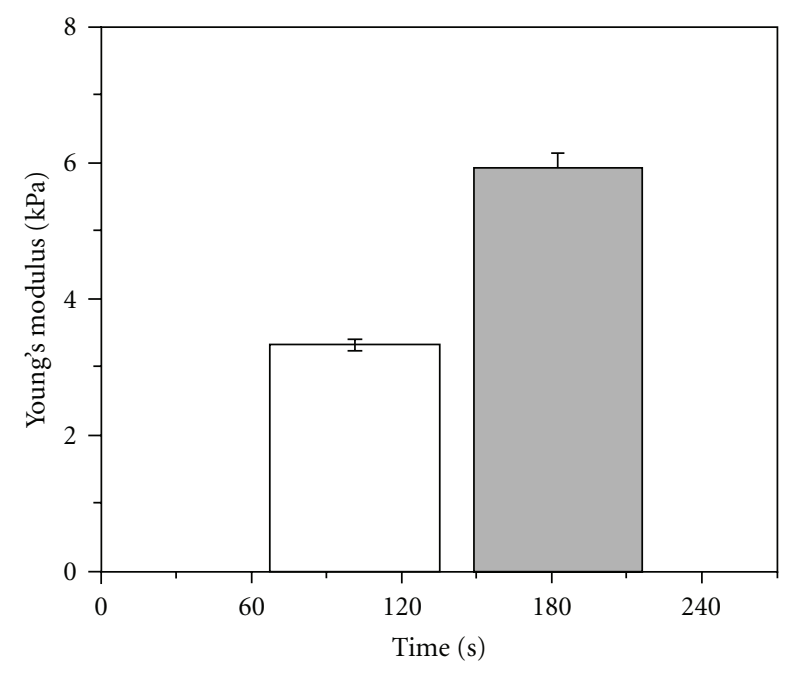

(a)

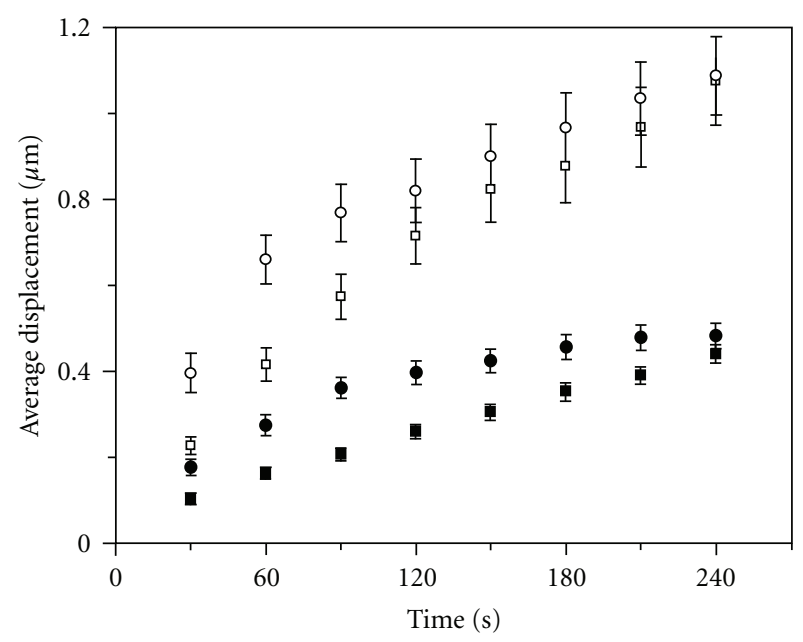

(b)

FIGURE 5: Cellular Young's modulus and average IF displacement as a function of time. (a) The average Young's modulus between elongated (white bar) and flat (grey bar) cells is $3.3 \pm 0.1$ and $5.9 \pm 0.2 \mathrm{kPa}$, respectively $\left(P=1 \times 10^{-9}\right)$. (b) The average displacement of nodes in a photobleached grid on the IF CSK in flat (black symbols) and elongated cells (white symbols) during a $20 \mathrm{nN}$ indentation (circles) and under control conditions (squares) plotted as a function of time. For elongated and flat cells there is a statistically significant increase in displacement at $t=30 \mathrm{sec}$ after force application $(P<0.001)$. However, for elongated cells this statistically significant difference disappears by $t=90 \mathrm{sec}$ $(P>0.05)$. For flat cells this difference disappears by $t=120 \mathrm{sec}$ $(P>0.05)$.

constant over time. Elongated and flat cells respond to force with a statistically significant increase in displacement immediately (within $30 \mathrm{sec}$ ) in response to indentation ( $P<0.001$ for both cases $)$, followed by slowly increasing displacement over the rest of the measurement time. It is interesting to note that although the displacement of IFs differs in its relationship at early times, both control and indented cells reach similar average displacements after 240 seconds ( $P>0.05$ for both conditions).

The displacement magnitude was plotted as a heat map in order to visualize the response of the whole cell (Figure 6). In control cells, we observed that natural movement of the IF CSK occurs throughout the cell over time. Elongated cells often displayed large displacement magnitudes, localized to a particular region of the cell. This phenomenon appeared regardless of force application and may possibly be due to the lack of an intact actin CSK as well as being poorly adhered to the underlying substrate. During force application we generally found that flat cells tended to display little displacement near the central longitudinal axis of the cells, but a large amount of displacement is observed in areas away from this axis (Figure 7). This displacement anisotropy was never observed in elongated cells. This is significant because although IFs are intact in both elongated and flat cells, only flat cells contain an intact actin CSK and FAs. Therefore, the interaction between IFs and a polarized actin CSK and/or strong surface interactions via FAs may account for the anisotropy observed in the deformation of IFs. The IF network is known for its integration with the other CSK elements in transmitting external forces from the extracellular matrix, through the FAs, into the cytosol and the nucleus [12]. As a result, the breakdown of other cytoskeletal elements such as FAs, as noted in Figure 3, may lead to the absence of IF deformation anisotropy.

\section{Discussion}

The goal of our study was to introduce a methodology employing simultaneous AFM-LSCM in order to study the realtime deformation of a specific component of the internal cell architecture in response to an applied external force. Photobleaching was first employed to create a grid pattern in cells expressing GFP-vimentin. Subsequently cells were exposed to an external force via the AFM that resulted in the deformation of IFs. By tracking the nodes of the photobleached grid we were able to spatially map the distribution of deformation throughout the cell over time. Two morphological populations of cells were investigated in this study: flat and elongated. It was observed that elongated cells have far fewer FAs than flat adherent cells. Thus it thought to reduce cell attachment to the substrate $[25,26]$, which, combined with the reduced Young's Modulus of the elongated cells, allows for increased deformation of the IF CSK.

The plot of the average displacement of IFs in control cells revealed that the IF CSK is constantly moving and evolving over time resulting in an apparent displacement of the IFs from their initial positions. However, in response to an externally applied force, the cells displayed a large increase immediately after indentation, followed by gradual increases in displacement eventually becoming similar to the control. In other words, in the absence of large, externally applied forces, the IF CSK reveals a constant rate of movement from the process of remodeling and tensions generated within the cell $[6,28]$. Interestingly, while the difference between the average displacement of vimentin in flat cells 


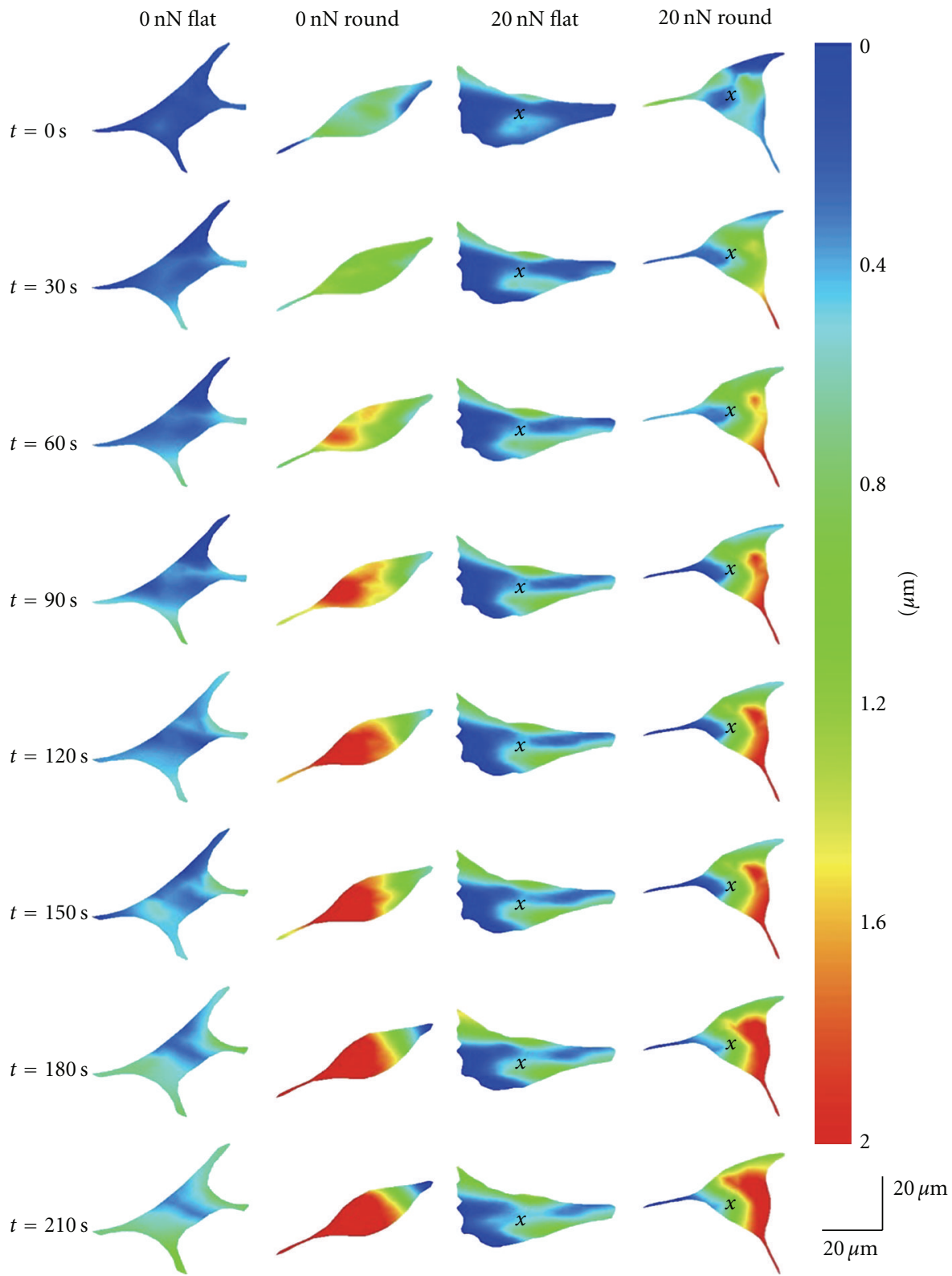

FIGURE 6: Colour contour maps revealing local displacement of IFs in both elongated and flat cells through a period of 210 sec under control and mechanically stimulated conditions. Cells that were indented by the AFM have the location of indentation (the centre of the nucleus) marked by an " $x$ ". Elongated cells tend to display large magnitude displacements, often localized towards one part of the cell, regardless of force application. Flat cells tend to display smaller (compared to elongated cells) and anisotropic IF movements in response to force application.

diminished at $t=240 \mathrm{sec}$, the difference in elongated cells diminished much earlier at $t=90 \mathrm{sec}$. This is likely due to the fact that the control elongated cells have an illformed actin CSK and few FAs making them unable to resist the movement of the IF CSK. Due to the reduced Young's modulus of elongated cells, it is also reasonable that the same applied force should lead to an increased initial displacement. On the other hand, flat cells possess a well-defined actin CSK and many FAs. This resulted in an anisotropic IF displacement profile. In response to force, the IF CSK tended to move away from the longitudinal axis of the cell along which actin stress fibres generally tend to polarize.

Our study has utilized a novel approach to studying force transmission occurring within a cell. Extracellular force was delivered to living cells in a controlled fashion while a combination of LSCM and patterned photobleaching was employed to quantify the magnitude and direction of force transmission. However, there are some limitations of the current study which limit the extent to which more generalized conclusions can be drawn about the biology of the IF CSK. These will be addressed in the future; however, the 


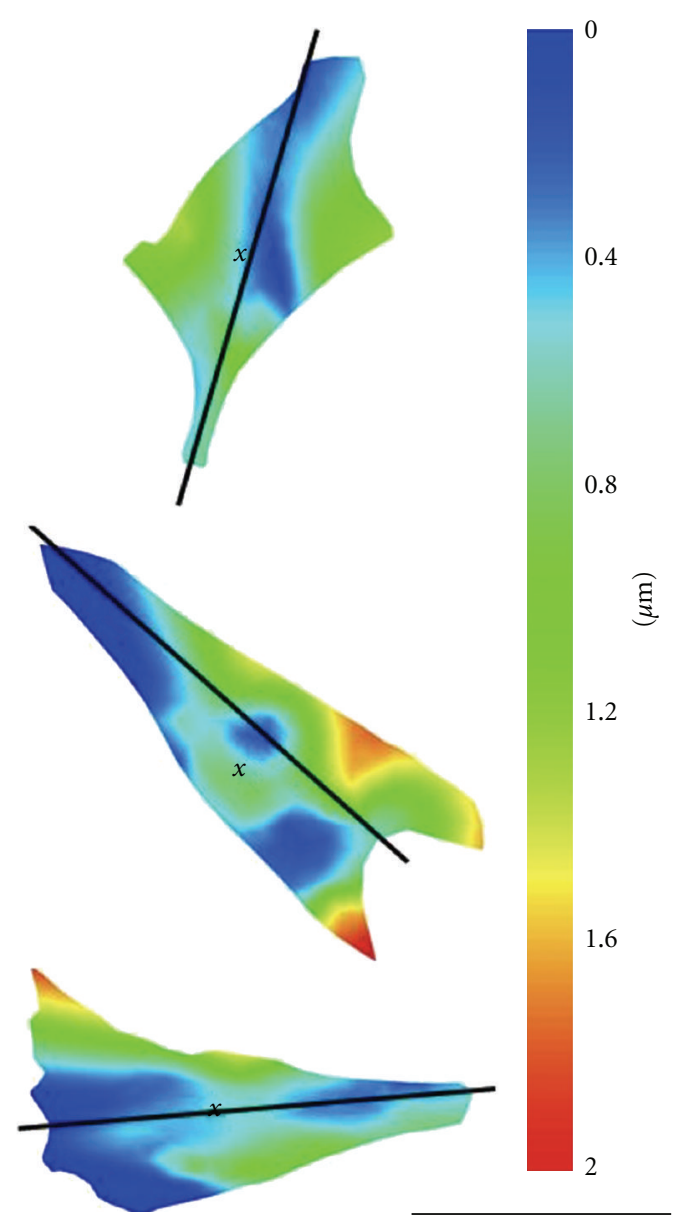

Figure 7: Anisotropic deformation of the IF CSK. Contour maps of three flat cells experiencing a $20 \mathrm{nN}$ indentation, with the central long axis of the cell labeled with a black line and the location of the AFM indentation labeled with an " $x$ ". IF deformation tended to be focused away from the central longitudinal axis. Scale bar $=30 \mu \mathrm{m}$.

methodology remains highly useful as a mechanism to study force transmission inside living cells. One clear limitation is the use of a GFP chimera protein and its interference with the endogenous IF CSK. Various chimeras have been developed and can alter basal mechanical properties, IF organization and cell dynamics in a variety of ways $[29,30]$. Secondly, the IF network is highly integrated with the rest of the structural components of the cell and cannot be considered to be acting completely independently of the rest of the CSK and cannot be considered in isolation. Thirdly, the physiological state of the elongated fibroblasts in this study is not well defined. These are all important issues which require further study and can be addressed with the use of cell lines expressing a variety of fluorescently labeled IF proteins, the use of specific anticytoskeletal drugs, and/or targeted siRNA. However, the methodology presented in this study remains a useful tool to quantify the structural response of the cytoarchitecture to mechanical stimulation.

IFs are a crucial component of the cell in both maintaining the integrity of the CSK structure as well as transmitting forces from the ECM into the cytosol. In this study, the displacement of the IF CSK as a whole was studied in response to deformation via indentation using the AFM. We have found that there is a major difference in the average displacement with respect to time between cells exhibiting a flat morphology and those exhibiting an elongated morphology. For both cases of elongated and flat cells, the indented cells show a large increase in the displacement of IFs over control cells immediately following indentation, but the overall average displacement of the IF CSK after $240 \mathrm{sec}$ of constant force application was statistically similar. This indicates that externally applied forces to the cell are rapidly transmitted throughout the IF CSK which rapidly dissipates the force towards baseline levels. The IF CSK appears to be imparting the cell with the ability to maintain its structural integrity in response to the sudden application of large external forces. IFs are highly dynamic and constantly altering their configuration, despite the absence of externally applied forces. Locally, the displacement of the IF CSK is highly anisotropic in flat cells, where the magnitudes of displacement are highest at areas furthest away from the central longitudinal axis. This is most likely a result of interactions between IFs and other cytoskeletal elements such as actin and FAs. This dynamic behaviour of the IF CSK is likely highly important to its function in maintaining the integrity of the cell in response to physical stress as well as in integrating mechanical signaling pathways mediated by the actin and MT CSK.

\section{Acknowledgments}

This study was funded by the Natural Sciences and Engineering Research Council (NSERC) Discovery Grant, an NSERC Discovery Accelerator Supplement and a Canada Research Chair (CRC) Operating Grant. J. Wang acknowledges support from the NSERC CREATE program for a summer fellowship. A. E. Pelling. gratefully acknowledges the support of the Canada Research Chairs (CRC) program and an Early Researcher Award from the Province of Ontario.

\section{References}

[1] H. Huang, R. D. Kamm, and R. T. Lee, "Cell mechanics and mechanotransduction: pathways, probes, and physiology," American Journal of Physiology, vol. 287, no. 1, pp. C1-C11, 2004.

[2] A. J. Engler, M. A. Griffin, S. Sen, C. G. Bönnemann, H. L. Sweeney, and D. E. Discher, "Myotubes differentiate optimally on substrates with tissue-like stiffness: pathological implications for soft or stiff microenvironments," The Journal of Cell Biology, vol. 166, no. 6, pp. 877-887, 2004.

[3] A. J. Engler, S. Sen, H. L. Sweeney, and D. E. Discher, "Matrix elasticity directs stem cell lineage specification," Cell, vol. 126, no. 4, pp. 677-689, 2006.

[4] I. Andricioaei, A. Goel, D. Herschbach, and M. Karplus, "Dependence of DNA polymerase replication rate on external forces: a model based on molecular dynamics simulations," Biophysical Journal, vol. 87, no. 3, pp. 1478-1497, 2004. 
[5] N. Wang, J. P. Butler, and D. E. Ingber, "Mechanotransduction across the cell surface and through the cytoskeleton," Science, vol. 260, no. 5111, pp. 1124-1127, 1993.

[6] J. Bertaud, Z. Qin, and M. J. Buehler, "Intermediate filamentdeficient cells are mechanically softer at large deformation: a multi-scale simulation study," Acta Biomaterialia, vol. 6, no. 7, pp. 2457-2466, 2010.

[7] Z. Qin, L. Kreplak, and M. J. Buehler, "Hierarchical structure controls nanomechanical properties of vimentin intermediate filaments," PLoS One, vol. 4, no. 10, Article ID e7294, 2009.

[8] Z. Qin, L. Kreplak, and M. J. Buehler, "Nanomechanical properties of vimentin intermediate filament dimers," Nanotechnology, vol. 20, no. 42, Article ID 425101, 2009.

[9] G. W. Brodland and R. Gordon, "Intermediate filaments may prevent buckling of compressively loaded microtubules," Journal of Biomechanical Engineering, vol. 112, no. 3, pp. 319-321, 1990.

[10] K. J. Green, M. Böhringer, T. Gocken, and J. C. R. Jones, "Intermediate filament associated proteins," Advances in Protein Chemistry, vol. 70, pp. 143-202, 2005.

[11] S. D. Georgatos and G. Blobel, "Lamin B constitutes an intermediate filament attachment site at the nuclear envelope," The Journal of Cell Biology, vol. 105, no. 1, pp. 117-125, 1987.

[12] A. J. Maniotis, C. S. Chen, and D. E. Ingber, "Demonstration of mechanical connections between integrins, cytoskeletal filaments, and nucleoplasm that stabilize nuclear structure," Proceedings of the National Academy of Sciences of the United States of America, vol. 94, no. 3, pp. 849-854, 1997.

[13] P. A. Janmey, U. Euteneuer, P. Traub, and M. Schliwa, "Viscoelastic properties of vimentin compared with other filamentous biopolymer networks," The Journal of Cell Biology, vol. 113, no. 1, pp. 155-160, 1991.

[14] L. Kreplak and D. Fudge, "Biomechanical properties of intermediate filaments: from tissues to single filaments and back," BioEssays, vol. 29, no. 1, pp. 26-35, 2007.

[15] T. Ackbarow, D. Sen, C. Thaulow, and M. J. Buehler, "Alphahelical protein networks are self-protective and flaw-tolerant," PLoS One, vol. 4, no. 6, Article ID e6015, 2009.

[16] G. Binnig, C. F. Quate, and C. Gerber, "Atomic force microscope," Physical Review Letters, vol. 56, no. 9, pp. 930-933, 1986.

[17] H. Haga, S. Sasaki, K. Kawabata, E. Ito, T. Ushiki, and T. Sambongi, "Elasticity mapping of living fibroblasts by AFM and immunofluorescence observation of the cytoskeleton," Ultramicroscopy, vol. 82, no. 1-4, pp. 253-258, 2000.

[18] M. Horton, G. Charras, C. Ballestrem, and P. Lehenkari, "Integration of atomic force and confocal microscopy," Single Molecules, vol. 1, no. 2, pp. 135-137, 2000.

[19] P. P. Lehenkari, G. T. Charras, S. A. Nesbitt, and M. A. Horton, "New technologies in scanning probe microscopy for studying molecular interactions in cells," Expert Reviews in Molecular Medicine, vol. 2, no. 2, pp. 1-19, 2000.

[20] P. Kunda, A. E. Pelling, T. Liu, and B. Baum, "Moesin controls cortical rigidity, cell rounding, and spindle morphogenesis during mitosis," Current Biology, vol. 18, no. 2, pp. 91-101, 2008.

[21] A. E. Pelling, F. S. Veraitch, C. P. K. Chu, C. Mason, and M. A. Horton, "Mechanical dynamics of single cells during early apoptosis," Cell Motility and the Cytoskeleton, vol. 66, no. 7, pp. 409-422, 2009.

[22] P. P. Lehenkari, G. T. Charras, A. Nykänen, and M. A. Horton, "Adapting atomic force microscopy for cell biology," Ultramicroscopy, vol. 82, no. 1-4, pp. 289-295, 2000.
[23] A. Kölsch, R. Windoffer, T. Würflinger, T. Aach, and R. E. Leube, "The keratin-filament cycle of assembly and disassembly," Journal of Cell Science, vol. 123, no. 13, pp. 2266-2272, 2010.

[24] R. Matzke, K. Jacobson, and M. Radmacher, "Direct, highresolution measurement of furrow stiffening during division of adherent cells," Nature Cell Biology, vol. 3, no. 6, pp. 607610, 2001.

[25] H. Ngu, Y. Feng, L. Lu, S. J. Oswald, G. D. Longmore, and F. C. P. Yin, "Effect of focal adhesion proteins on endothelial cell adhesion, motility and orientation response to cyclic strain," Annals of Biomedical Engineering, vol. 38, no. 1, pp. 208-222, 2010.

[26] A. P. Zhu and N. Fang, "Adhesion dynamics, morphology, and organization of 3T3 fibroblast on chitosan and its derivative: the effect of O-carboxymethylation," Biomacromolecules, vol. 6, no. 5, pp. 2607-2614, 2005.

[27] R. Kalluri and M. Zeisberg, "Fibroblasts in cancer," Nature Reviews Cancer, vol. 6, no. 5, pp. 392-401, 2006.

[28] M. Yoon, R. D. Moir, V. Prahlad, and R. D. Goldman, "Motile properties of vimentin intermediate filament networks in living cells," The Journal of Cell Biology, vol. 143, no. 1, pp. 147-157, 1998.

[29] M. Plodinec, M. Loparic, R. Suetterlin, H. Herrmann, U. Aebi, and C.-A. Schoenenberger, "The nanomechanical properties of rat fibroblasts are modulated by interfering with the vimentin intermediate filament system," Journal of Structural Biology, vol. 174, no. 3, pp. 476-484, 2011.

[30] C. L. Ho, J. L. Martys, A. Mikhailov, G. G. Gundersen, and R. K. H. Liem, "Novel features of intermediate filament dynamics revealed by green fluorescent protein chimeras," Journal of Cell Science, vol. 111, part 13, pp. 1767-1778, 1998. 

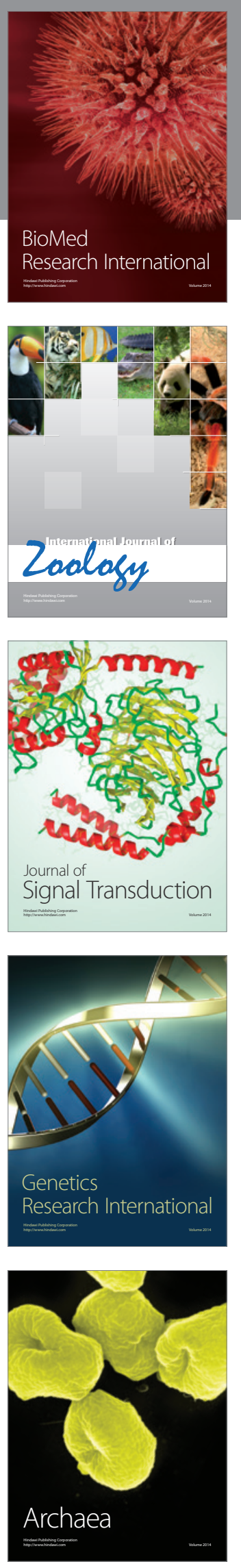
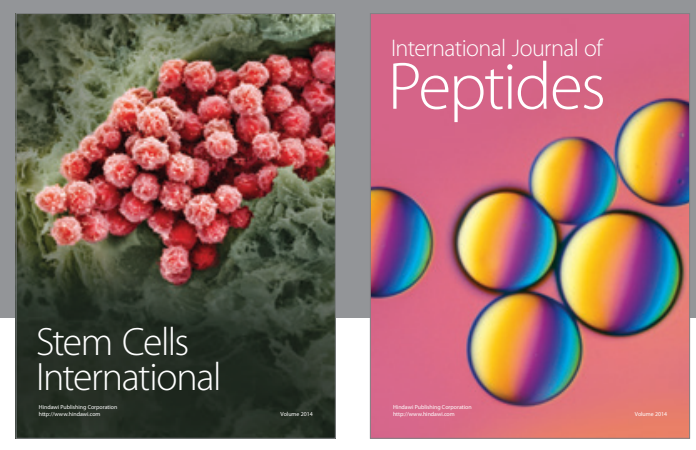

Submit your manuscripts at

http://www.hindawi.com
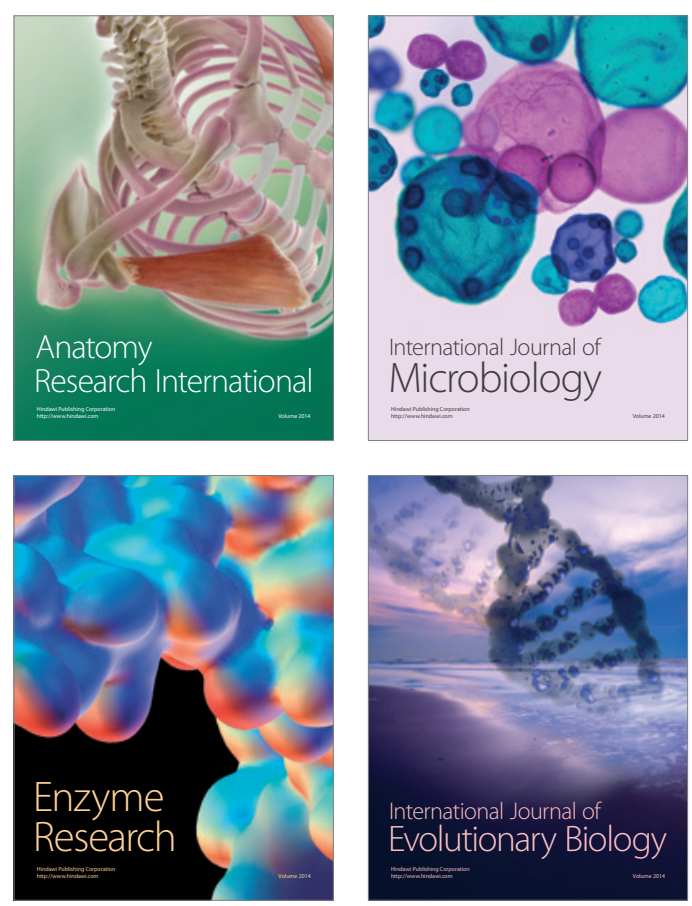
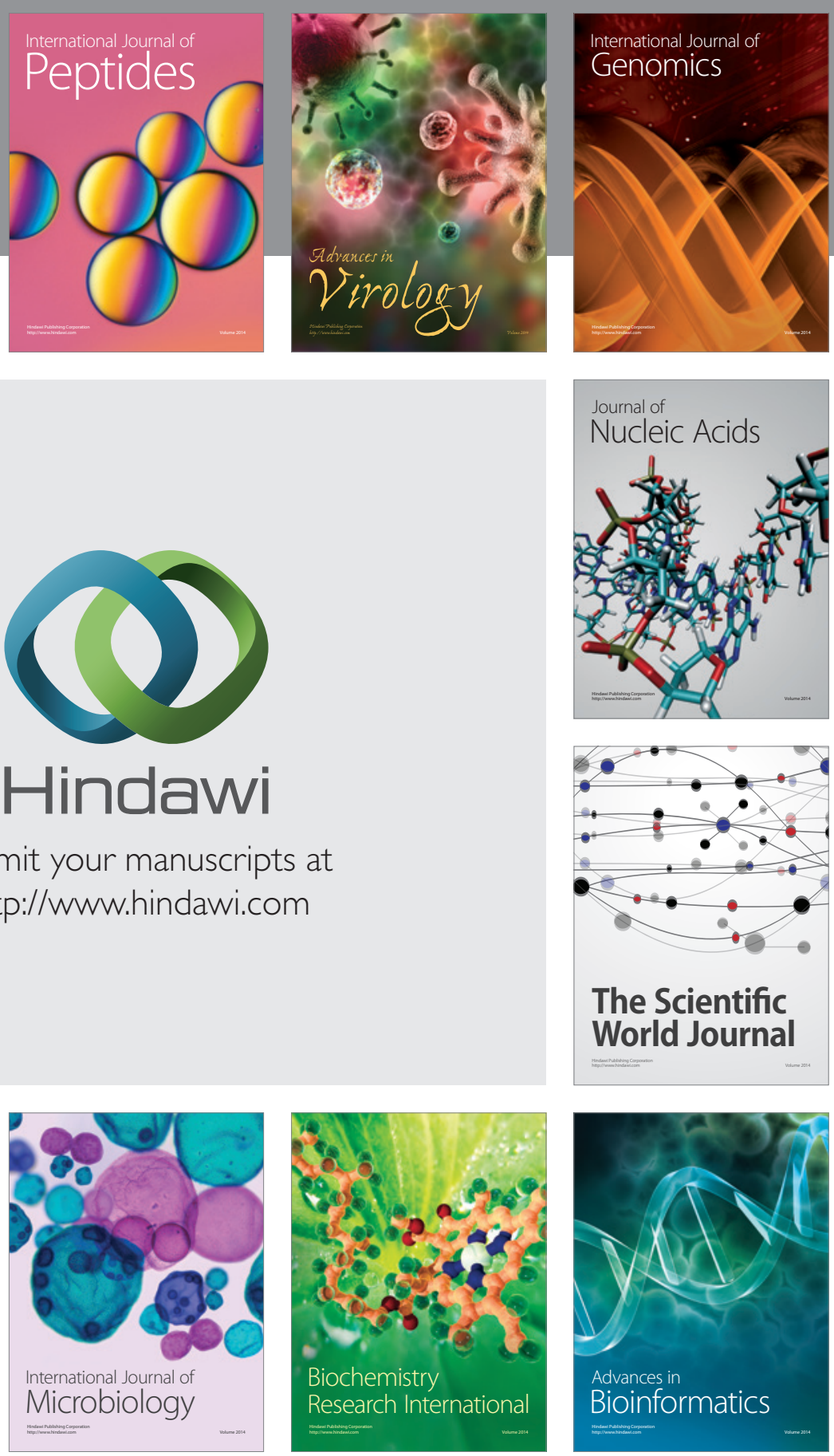

The Scientific World Journal
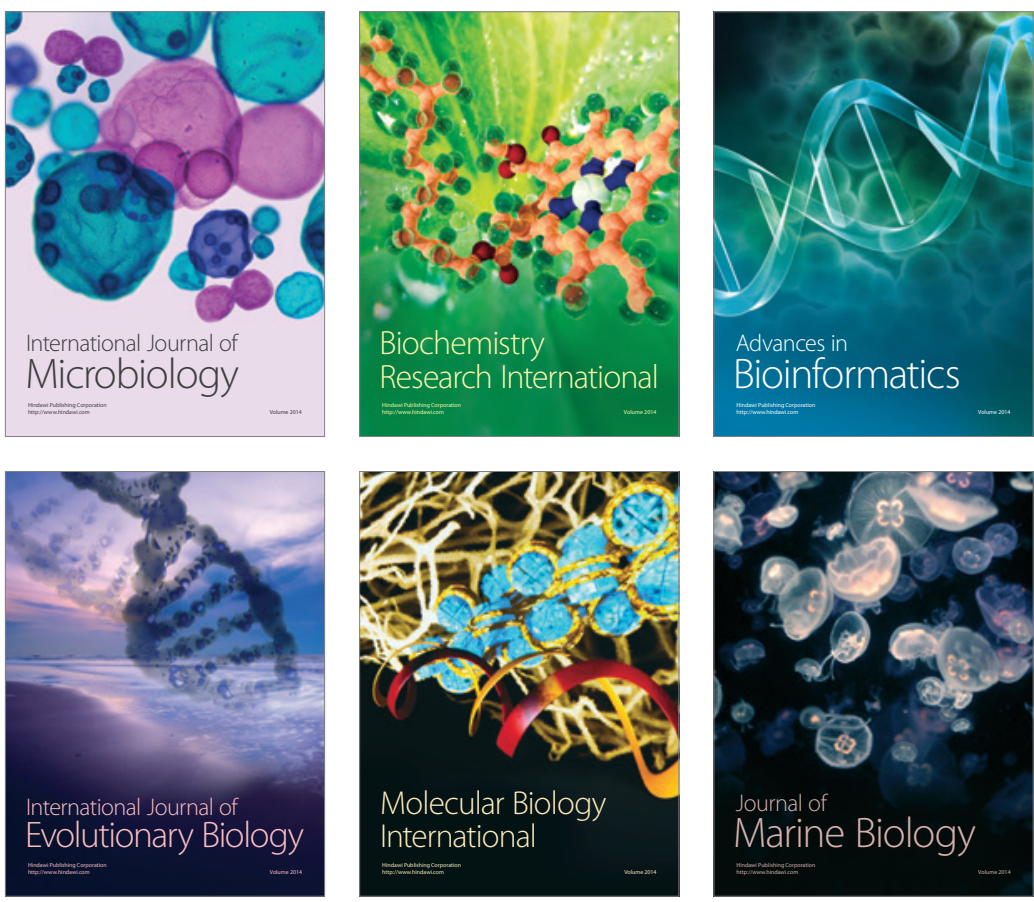